\title{
Identification and analysis of in planta expressed genes of Magnaporthe oryzae
}

Soonok Kim², Jongsun Park', Sook-Young Park' , Thomas K Mitchell², Yong-Hwan Lee ${ }^{1 *}$

\begin{abstract}
Background: Infection of plants by pathogens and the subsequent disease development involves substantial changes in the biochemistry and physiology of both partners. Analysis of genes that are expressed during these interactions represents a powerful strategy to obtain insights into the molecular events underlying these changes. We have employed expressed sequence tag (EST) analysis to identify rice genes involved in defense responses against infection by the blast fungus Magnaporthe oryzae and fungal genes involved in infectious growth within the host during a compatible interaction.

Results: A cDNA library was constructed with RNA from rice leaves (Oryza sativa cv. Hwacheong) infected with M. oryzae strain KJ201. To enrich for fungal genes, subtraction library using PCR-based suppression subtractive hybridization was constructed with RNA from infected rice leaves as a tester and that from uninfected rice leaves as the driver. A total of 4,148 clones from two libraries were sequenced to generate 2,302 non-redundant ESTs. Of these, 712 and 1,562 ESTs could be identified to encode fungal and rice genes, respectively. To predict gene function, Gene Ontology (GO) analysis was applied, with 31\% and 32\% of rice and fungal ESTs being assigned to GO terms, respectively. One hundred uniESTs were found to be specific to fungal infection EST. More than 80 fulllength fungal cDNA sequences were used to validate $a b$ initio annotated gene model of $M$. oryzae genome sequence.

Conclusion: This study shows the power of ESTs to refine genome annotation and functional characterization. Results of this work have advanced our understanding of the molecular mechanisms underpinning fungal-plant interactions and formed the basis for new hypothesis.
\end{abstract}

\section{Background}

Rice blast, caused by Magnaporthe oryzae, is one of the most devastating diseases in rice growing regions worldwide, causing $11-15 \%$ yield loss annually [1]. Genetic tractability as well as economic importance makes this disease a model pathosystem to understand plantmicrobe interactions. The genome sequences of both organisms are available [2-4], and both forward and reverse genomic studies to understand molecular mechanisms for pathogenesis on a genome scale have been undertaken [5]. Understanding the precise molecular mechanisms of infection will facilitate design of novel control strategies.

\footnotetext{
* Correspondence: yonglee@snu.ac.kr

'Department of Agricultural Biotechnology, Center for Fungal Pathogenesis, Center for Agricultural Biomaterials and Center for Fungal Genetic Resources, Seoul National University, Seoul 151-921, Korea
}

The process of M. oryzae infection starts when a conidium lands on the rice leaf surface. After germination by hydration, an appressorium develops at the tip of the germ tube, from which a penetration peg emerges to penetrate the cuticle layer into the rice cell using mechanical force. Within the plant cell, the fungus faces two different fates. In an incompatible interaction, resistance gene products recognize corresponding avirulence gene products from the invading pathogen and invoke a series of defense responses to restrict pathogen growth [6]. In a compatible interaction, however, the host plant mobilizes defense responses much later, resulting in visible coalescing lesions. From these expanding lesions, a conidiophore emerges, releasing tens of thousands of new conidia able to start a second round of infection. In this respect, the gene products of the pathogen expressed during infectious growth may play roles as pathogenicity factors required for evasion of the host's 
defense responses and successful colonization. This theory is supported by our previous pilot study in which analysis of in planta expressed genes identified by EST sequencing of an M.oryzae infected rice cDNA library [7]. By sequencing 511 randomly selected cDNA clones, 72 of 293 ESTs could be assigned as fungal genes with sequence similarity to NCBI entries. Among them, $M H P 1$, encoding a Class II hydrophobin, was preferentially expressed during infectious growth and was involved in full virulence of rice blast fungus, acting in the late stages of infection [8]. We also identified novel pathogenesis-related protein genes that are upregulated in rice after pathogen infection $[9,10]$. Jantasuriyarat et al. [11] performed large-scale analysis of ESTs during M.oryzae/rice interactions at the early stage of infection. Plant defense genes were well represented in this study. However, fungal genes involved in interactions with the host were scarce, primarily because they harvested infected tissue at 6 and $24 \mathrm{~h}$ after inoculation, at which time fungal spores had just started to penetrate. Genome-wide transcriptome analyses have also been conducted on this fungus within the last few years through the use of EST, SAGE, and RL-SAGE [7,11-16]. Most of these studies focused on pre-penetration stages, such as conidiation and appressorium formation, and on growth in a variety of in vitro conditions, including complete medium, minimal medium, nitrogen starvation, and rice cell wall medium. From these global efforts, 28,682 EST sequences comprising 8,821 uniESTs of $M$. oryzae are registered in the dbEST of NCBI, which covers about $80 \%$ of the genes electronically annotated for this fungus [2]. However, our knowledge of the molecular basis of the establishment of disease and proliferation within the plant cells remains limited.

During recent years, studies aimed at cataloging pathogen genes expressed during interactions with their host have been conducted in many pathosystems. Largescale EST analysis with cDNA libraries from infected plant tissues has been used for this purpose [17]. More than 1,000 fungal genes could be identified out of 1,869 EST sequences from senescent leaves (21-25 days after inoculation) with lesions containing visible pycnidia. However, few fungal genes could be recovered from Fusarium graminearum infected wheat leaves (86 of 3,546 uniESTs) [18], from Brassica napus stem segments with expanding lesions caused by Sclerotinia sclerotiorum (52 of 767 uniESTs) [19], from wheat roots infected by take-all fungus (9 of 114 ESTs) [20], or $M$. oryzae infected rice leaves (4 of 13,570 uniESTs) [11]. Low-level representation of fungal genes may be because they harvested infected tissues at early time points. Suppression subtractive hybridization was also applied to identify genes expressed during the pathogen's interaction with its host [20,21]. Broeker et al. [21] identified
81 Puccinia graminis f. sp. tritici genes by sequencing 454 random clones from rust-infected wheat plants subtracted from those of healthy leaves. In this study, we used two complementary approaches: EST analysis and suppression subtractive hybridization and subsequent sequencing to catalog genes expressed during rice- $M$. oryzae interactions at the genome level. 712 fungal genes were identified from 2,315 uniESTs and their putative functions were assessed with GO. We were able to identify 100 novel fungal genes specific to infection. cDNAs with full-length ORFs were used to correct $a b$ initio gene models annotated from genome sequences, and to analyze fungal splice site context.

\section{Results}

\section{Generation of infection ESTs}

We identified in planta expressed fungal genes by EST analysis of a M.oryzae-infected rice cDNA library in a previous study [7]. Seventy-two ESTs comprising 19 unigenes were assigned as fungal genes. To expand our knowledge of the molecular mechanisms underlying plant-microbe interactions, cataloging gene repertoires at a genome-wide level was carried out with two complementary strategies. First, an infection library (IL) was constructed with RNAs from leaves showing rapidly expanding lesions. cDNAs more than $0.5 \mathrm{~kb}$ in size after gel filtration were used for library construction. The titer of the primary library was $1.85 \times 10^{7} \mathrm{pfu} / \mu \mathrm{g}$ vector, and the insert size ranged from 0.5 to $2.0 \mathrm{~kb}$. Sequencing of 1,976 randomly selected cDNAs generated 1,539 non-redundant ESTs, of which 654 ESTs formed 217 contigs and 1,322 ESTs remained as singletons (Table 1). Second, to enrich for fungal genes expressed during colonization and propagation within the plant cells, as well as plant genes induced by the invading pathogen, we used suppression subtractive hybridization technology [22]. cDNAs synthesized with RNA from uninfected rice leaves were used as the driver, while those from heavily infected rice leaves were used as the tester. A total of 2,259 subtracted clones were sequenced, generating 963 nonredundant ESTs. In the subtraction library (SL), the redundancy of the sequences was relatively high, with 1,722 clones assembled into 426 contigs. When the sequences from two libraries were analyzed together, all the 4,235 ESTs fell into 2,302 non-redundant ESTs, in which 2,538 ESTs consisted of 605 contigs and 1,697 ESTs remained as singletons (Table 1). About $69 \%$ of these 2,302 nrESTs had high or moderate sequence similarity to the entries of the non-redundant database in NCBI through BLAST search.

The most frequently represented sequence was contig203, which showed sequence similarity to elongation factor 1- $\alpha$ of the dimorphic fungus Ajellomyces capsulatus [23]. It occurred 76 times from SL and five times 
Table 1 Summary of ESTs generated from infection and subtractrion libraries

\begin{tabular}{lccc}
\hline \multicolumn{1}{c}{ Library } & $\mathbf{I L}^{\mathbf{1}}$ & $\mathbf{S L}^{\mathbf{2}}$ & Total \\
\hline Number of sequences analyzed & 1,976 & 2,259 & 4,235 \\
Number of contigs & 217 & 426 & 605 \\
Non-redundant EST & 654 & 1,722 & 2,538 \\
Number of singletons & 1,322 & 537 & 1,697 \\
Number of cDNAs contained in contigs & 1,539 & 963 & 2,302
\end{tabular}

${ }^{1} \mathrm{IL}$ means infection library constructed in UNI-ZAP XR vector.

${ }^{2} \mathrm{SL}$ means subtraction library in which RNA from $M$. oryzae infected rice

leaves was used as tester and that from uninfected leaves as driver.

from IL. Contig226 was sequenced 63 times only from SL, and Contig200, showing sequence similarity to senescence-associated protein of Pisum sativum [24], appeared 29 times in SL and once in IL. The repertoire of highly redundant clones differed between the two libraries (Additional file 1, Table S1). In IL, genes involved in photosynthesis, such as RuBisCo activase (Contig 526) and RuBisCo small subunit C (Contig 549), and plant defense-related genes, such as metallothionein (Contig 496) and pathogenesis-related protein 1 (Contig 597), were included in the 10 most redundant contigs. Only two contigs (Contig 310 and Contig 370) of the 10 most redundant contigs were considered to be of fungal origin. However, fungal genes made up $50 \%$ of the most redundant 10 contigs of SL. Additionally, after subtracting, genes involved in photosynthesis, defense-related genes including senescence-associated protein (Contig 200), probenazole-induced protein (Contig 173), and metallothionein I (Contig 153) were the most abundant sequences.

\section{Annotation of the origin of genes}

After initial analysis, the origin of ESTs was predicted. We took advantage of the most recent genome assembly of rice [3,4] and M.oryzae [2], as well as the deep cDNA sequence resources available for the host and pathogen. EST sequences were initially mapped on the genome sequence of both organisms, with 1,342 ESTs (773

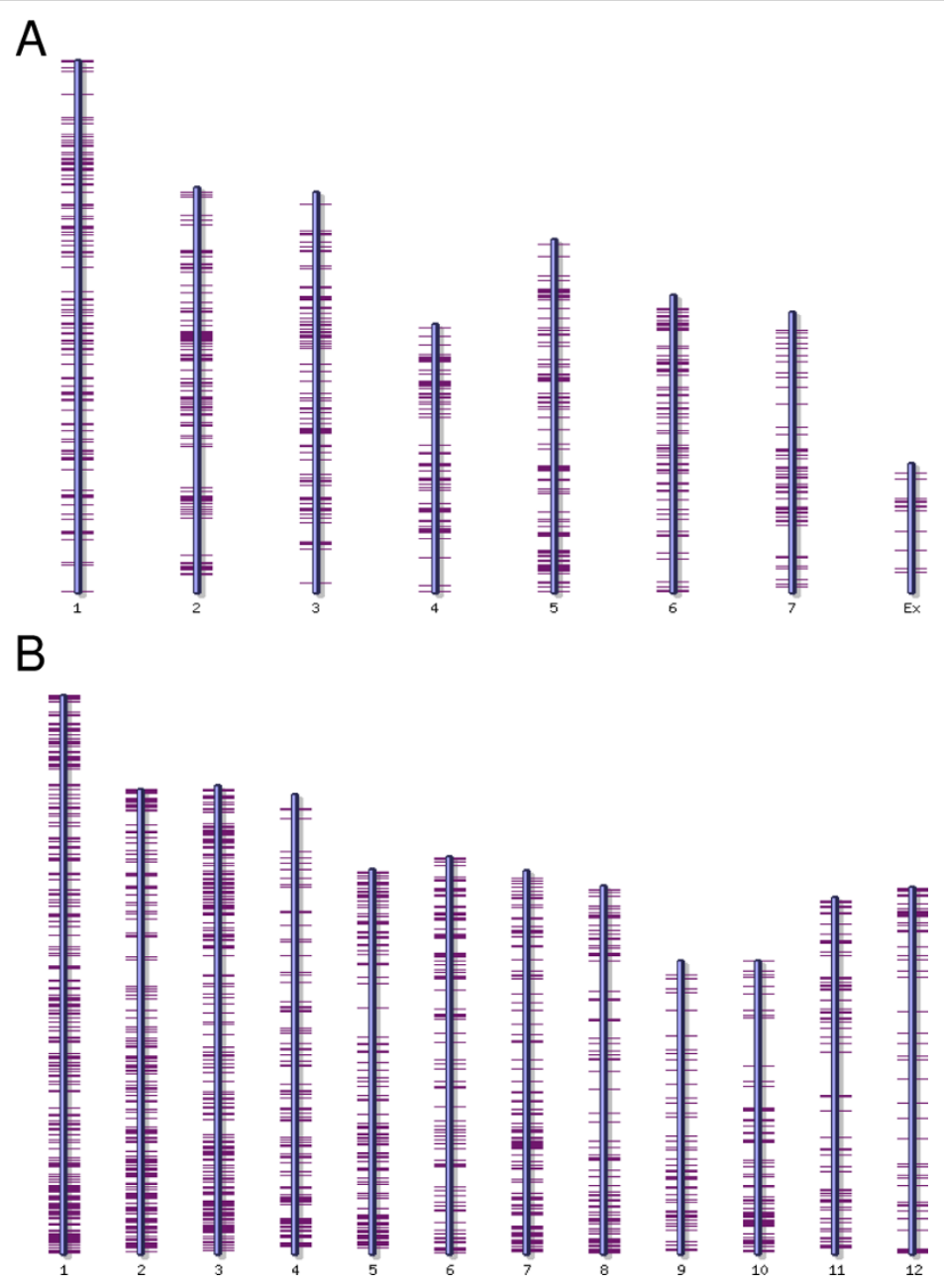

Figure 1 Genome wide distribution of infection ESTs. EST sequences were mapped to M. oryzae (A) or rice (B) chromosomes. 
Table 2 Classification of 2,315 uniESTs according to origin of the sequences

\begin{tabular}{lccc}
\hline & Fungal genes & Rice genes & Unidentified \\
\hline UniESTs (\%*) & $712(30.9)$ & $1,562(67.9)$ & $28(1.2)$ \\
Singletons & 468 & 1,205 & 24 \\
Contigs (No. ESTs) & $244(1,193)$ & $357(1,327)$ & $4(48)$ \\
\hline Infection library specific & $327(373)$ & $1,038(1,210)$ & $87(568)$ \\
Both library & $75(587)$ & $437(754)$ & \\
Subtraction library specific & $310(701)$ &
\end{tabular}

The origin of sequences were determined by the searches of rice (Indica type) and $M$. oryzae (strain 70-15) draft genome sequence, dbEST with BLASTN algorithm at expect value of $<\mathrm{e}^{-30}$ and NCBI non-redundant protein db with BLASTX algorithm $\left(<\mathrm{e}^{-5}\right)$.

* Proportion was calculated based on 2,302 uniESTs.

UniESTs) being mapped to the fungal genome and 3,817 $(1,546)$ to the rice genome (Fig. 1). Some of the ESTs were mapped to both genome sequences with the criteria we used $(\mathrm{e}<1 \mathrm{e}-5)$. Therefore, BLASTN data against $8,821 \mathrm{nrEST}$ sequences from nine different libraries of $M$. oryzae [12], and 32,127 rice full-length cDNA (fl cDNA) sequences [25] and BLASTX data against NCBI non-redundant protein database were further analyzed together to assign the origin of uniESTs. As a result, 2,302 uniESTs could be grouped into 712 fungal genes (31\%) and 1,562 plant genes (68\%; Table 2). There were still 28 uniESTs of unidentified origin due to the lack of sequence similarity to either organism. The fungal genes consisted of 468 singletons and 244 contigs from 1,193 ESTs, whereas the rice genes consisted of 1,205 singletons and 357 contigs from 1,327 ESTs. ESTs from each library were evaluated in terms of origin of the genes.
Of 1,539 uniESTs (1,976 ESTs) obtained from IL, 402 uniESTs (533 ESTs) could be assigned as fungal genes, which is $26.2 \%$ of the uniESTs (27\% of total ESTs). For the 963 uniESTs of SL, 385 uniESTs, comprising $40 \%$ of uniESTs, were found to be of fungal origin. The proportion of fungal genes increased to $50 \%$ when the raw EST sequences were taken into consideration, as 1,128 ESTs of 2,259 ESTs from SL were grouped as fungal genes. Of the 712 uniESTs of fungal origin, 327 uniESTs were IL-specific and 310 uniESTs were only found in SL. Seventy-five genes were sequenced from both libraries. Similarly, 437 uniESTs from SL were added to the 1,125 rice genes identified from IL. We then questioned whether the fungal genes could cluster in the genome by aligning to the current genome assembly. However, infection ESTs did not cluster, but were rather spread evenly throughout the genome (Fig. 1).
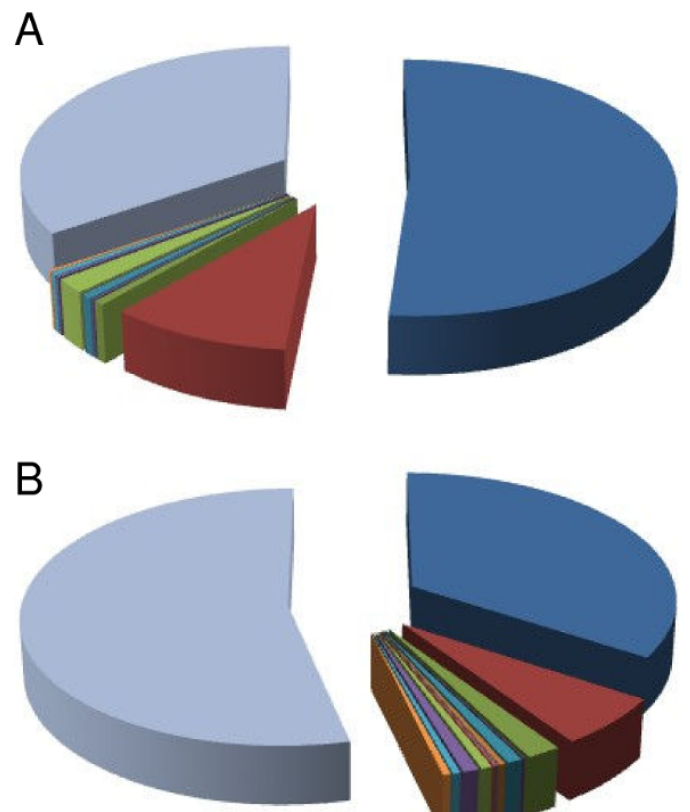

- metabolism
- cell growth and/or maintenance
- response to stress

- response to endogenous stimulus

- response to external stimulus

- homeostasis

- respiratory gaseous exchange

= photosynthesis

w cell communication

m cell death

$=$ development

= regulation of enzyme activity

m Unassigned

Figure 2 Gene Ontology (GO) annotation of fungal (A) and plant genes (B) expressed during their interaction. The ratio of hits to Biological Process is indicated. Fungal and rice ESTs were used to search matching ORFs from genome and full-length cDNA sequences, respectively. Translated ORF sequences were subjected to InterPro and GO analyses. 


\section{GO-based functional classification}

To assess the putative function of the ESTs, gene ontology (GO) based classification was conducted. Fungal and rice ESTs were translated in six frames, and the inframe ORF sequences were subjected to InterPro and GO annotation, with $42.4 \%$ (663) of rice ESTs and $58.1 \%$ (414) of fungal ESTs being assigned GO terms. In terms of biological process, 463 rice and 285 fungal genes were assigned; most were associated with metabolism, followed by cell growth and/or maintenance. Nine uniESTs encoding fungal genes were assigned to cell communications (GO: 0007154), which contained two class II hydrophobins (F204 and F233), four GTP-binding proteins (F0250 encoding small GTPase RAS2, F0253, F0265, and F0694), and an NADP-dependent oxidoreductase (F0064). For molecular function, 556 rice and 343 fungal ESTs were assigned, most of which possessed binding and catalytic activity. In the 208 rice and 169 fungal ESTs assigned to cellular components, 199 rice and 165 fungal ESTs were associated with the cell, and were especially distributed within the intracellular and membrane (Fig. 2).

Proteins assigned as extracellular were poorly represented in this analysis, although secretion proteins are thought to play important roles in host interactions $[26,27]$ and virulence $[8,28,29]$. Thus, we screened 2,302 uniESTs for signal peptides using SignalP3.0 [30]. Because most EST sequences did not bear a start codon, genomic resources were used. For fungal genes, $a b$ initio annotated protein sequences matched to infection ESTs were subjected to SignalP analysis. In the case of rice genes, where 32,127 full-length (fl-) cDNA sequences are available, fl cDNA sequences matched to infection ESTs were translated and in-frame amino acid sequences were screened with SignalP. As a result, 63 fungal genes and 246 rice genes of 2,302 uniESTs were believed to have a signal peptide. Among these were three hydrophobins (F204, F233 encoding MHP1, and F238 encoding MPG1), two of which have been identified as secreted and associated with the cell wall $[8,29]$. ESTs encoding spore coat proteins (F207) and cell wall degrading enzymes, such as chitinase3 (F037) and 1,4beta-D-glucan cellobiohydrolase (F298), were also identified.

\section{Search for novel genes uniquely or preferentially expressed during rice- $M$. oryzae interactions}

We questioned whether there were novel genes as yet unidentified because the condition used (i.e., late stage of infection) is uncommon. To identify novel genes preferentially expressed during rice- $M$. oryzae interaction, 2,302 uniESTs were compared with EST or cDNA sequences of each organism using BLAST. M. oryzae uniEST sequences $(8,820$ COGEME_EST $)$ were

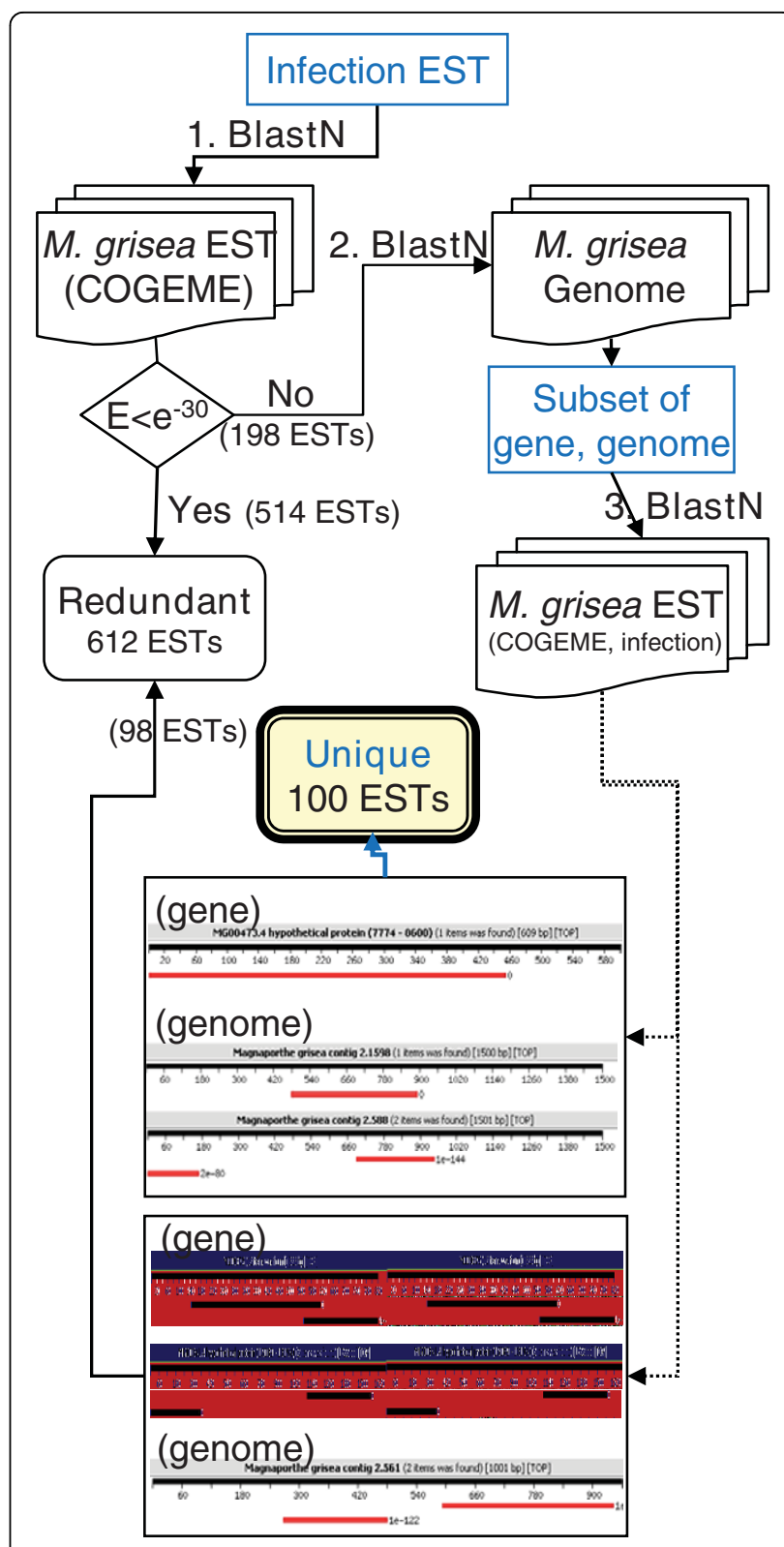

Figure 3 Process to search novel genes uniquely or preferentially identified during rice-blast fungus interactions

downloaded from COGEME phytopathogenic fungi and oomycete EST database (http://cogeme.ex.ac.uk). Rice full-length cDNA sequences $(32,127)$ were downloaded from the KOME (Knowledge-based Oryza Molecular Biological Encyclopedia: http://cdna01.dna.affrc.go.jp/ cDNA) website. For fungal genes, both COGEME EST and infectionEST collections were mapped on the $M$. oryzae genome, and the physical positions on the genome were compared between the two populations. Of the 712 putative fungal genes, 208 infectionESTs were not contained in the COGEME uniESTs. These EST sequences were further examined for sequences that 


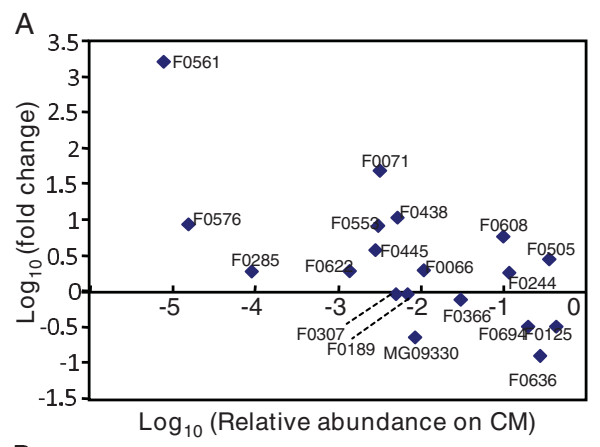

B

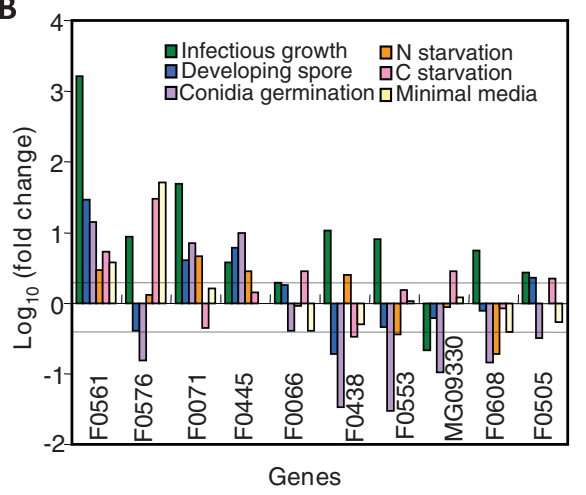

Figure 4 Expression of infection EST specific fungal genes analyzed by real-time RT-PCR. Relative abundance and fold changes were calculated as described in Experimental Procedures. Cyclophillin was used as an internal control. (A) Fold changes during infectious growth were compared relative abundance during growth in complete medium. (B) Fold changes during infectious growth, conidiation, conidial germination, and nutrient starvation conditions were depicted as log scale.

represent different segments of the same gene present in other libraries to determine if they were truly novel genes. In the case of genic regions represented by two or more ESTs, these ESTs were considered to be the same gene. If two or more ESTs matched to non-genic sequences, they were connected end to end or placed with gaps less than 400 nts, these ESTs were also considered to represent the same gene (Fig. 3). Through this analysis, we finally assigned 100 fungal uniESTs (14\%, 138 ESTs) as uniquely or preferentially expressed during infectious growth (Additional file 2, Table S2). Of these, 60 genes were not able to be assigned a putative function through the BLASTX search of NCBI nonredundant protein database. There were three groups of genes predicted to be functioning during direct interaction between the two organisms: genes involved in nutrient uptake and primary metabolism (F393, F415, F478, F561, F566, F575, and F597), cell wall processing (F287, F295, F305, and F541), and lipid metabolism (F125, F438, F553, and F636). Among these novel genes were two different members (F0438, MGG_05988 and
F0553, MGG_09994) for phosphatidic acid phosphatase (PAP), which is an enzyme that deposphorylates phosphatidic acid (PA) to generate diacylglycerol (DAG), both of which are known to be involved in lipid metabolism and intracellular signal transduction [31,32]. The remaining member (MGG_09330) was sequenced from the nitrogen-starved library [12]. A gene (F0066, MGG_04205) homologous to avenacinase, which detoxifies plant saponins to determine the host range, was also identified as expressed in planta. With a similar approach, 52 rice uniESTs did not match the rice EST sequences registered in the dbEST of NCBI. Most of them showed no sequence similarity to the non-redundant protein database, suggesting a unique role(s) during interactions with the pathogen.

\section{Expression profiles of infection-enriched genes}

Expression profiles of 20 infection EST-specific genes were analyzed by real-time RT-PCR. Fold changes (2$\Delta \Delta \mathrm{Ct}$ ) during infectious growth were analyzed in terms of relative abundance $\left(2^{-\Delta C t}\right)$ of transcripts during in vitro growth in complete media $(\mathrm{CM})$, compared with the cyclophilin (CYP1) expression level (Fig. 4A). Analyzed genes were grouped into three categories according to the expression level in CM. Expression level of six genes were 0.1 - to 0.45 fold that of CYP1 in CM; three were induced and three were repressed during infectious growth. Transcript levels of 10 genes were 0.1 to $3.0 \%$ that of CYP1 in CM; six of them were induced during in planta growth. Four genes were scarcely expressed in CM, but their expression was elevated in planta. Expression of nine genes upregulated more than twofold in planta was further examined during development and in nutrient conditions (Fig. 4B). Expression of F0561 encoding 3-phytase A was highly induced during infection and also induced more than twofold in all conditions tested. Expression of F0576 encoding aminopeptidase was co-up-regulated during infection, carbon starvation, and in minimal medium, while it was downregulated during conidia germination. F0071, with unknown function, and F0445, encoding ATP-dependent RNA helicase, shared their expression pattern and were co-up-regulated during infection, asexual sporulation, conidia germination, and nitrogen starvation. The expression pattern of F0066, showing sequence similarity to avenacinase (Gaeumannomyces graminis), and F0505, homologous to MADS-box homolog Umc1 (Ustilago maydis), was the same, with induction in planta, in developing spores, and during carbon starvation, and repression in germination and minimal media. F0608, with sequence similarity to yeast maintenance protein dam1, had specific up-regulation during growth within the plant cell. Three members of genes encoding phosphatidic acid phosphatase showed differential expression 


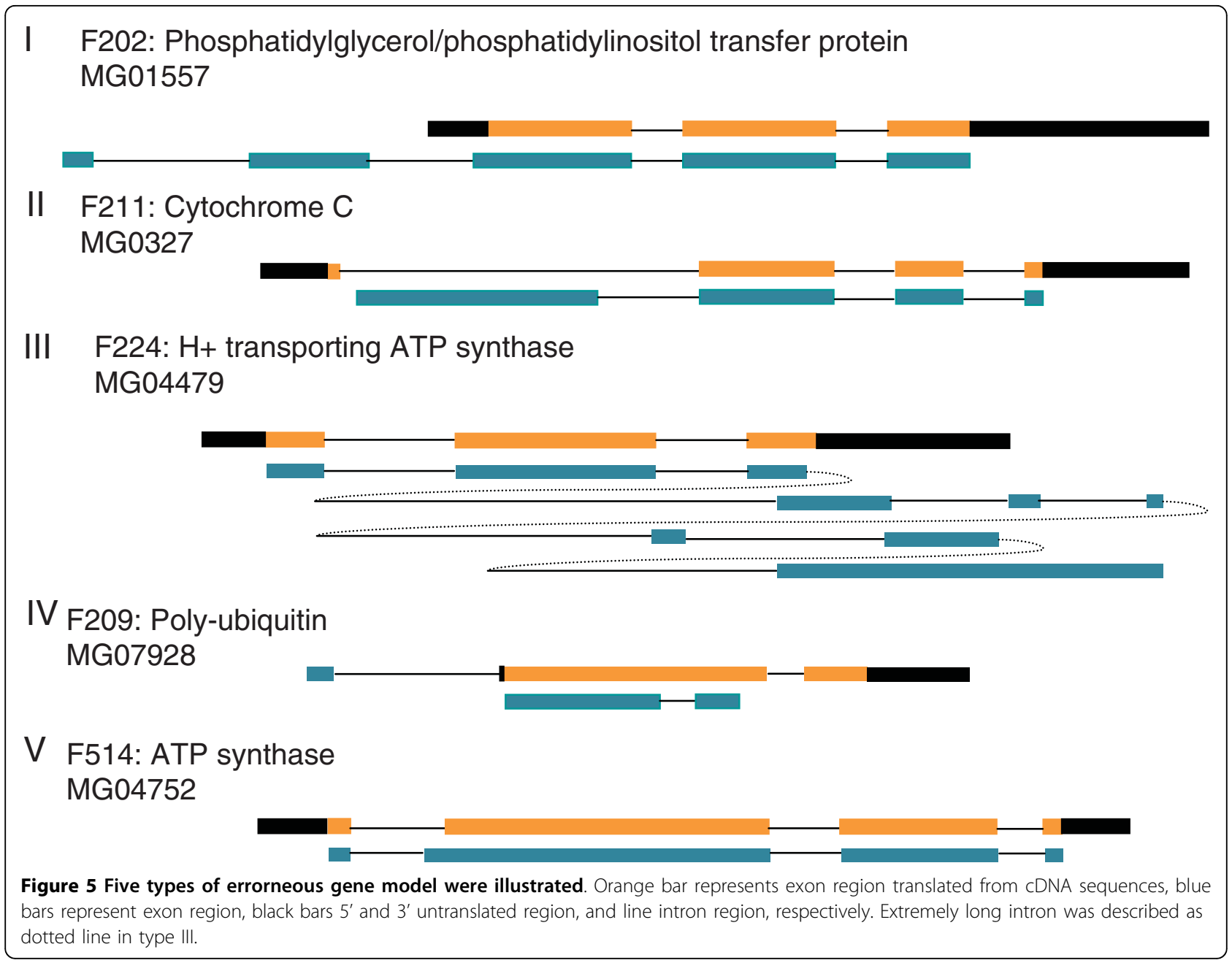

patterns: two of them (F0438 and F0553) were up-regulated during infection, but the other (MGG_09330) was down-regulated. F0438 was induced in nitrogen starvation, MGG_09330 in carbon starvation, and all three were down-regulated during conidiation and conidia germination.

\section{Use of ESTs in validation and improvement of genome annotation}

ESTs can complement automated genome annotation efforts [33]. 125 uniESTs showed no sequence similarity to $a b$ initio annotated gene sequences, but matched the genome assembly. These loci can now be re-annotated as genes. BLASTX results against the NCBI non-redundant protein database were carefully examined for ESTs that matched from a start codon to search for ESTs having full-length cDNA sequences. Among the 712 fungal genes, 83 uniESTs were found to have full-length ORFs through analysis of BLASTX results. These include genes uniquely identified from the infection EST, including genes encoding cytochrome P450 (F0125), co-chaperone
(F0132), and alcohol dehydrogenase (F0289) (Additional file 3, Table S3). Representative clones were retrieved, and entire inserts were sequenced using primer walking. It is possible that polymorphisms may be found by comparing the cDNA sequences to the genome, because our cDNA sequences were derived from $M$. oryzae strain KJ201, not strain 70-15, which was used for genome sequencing; however, on average, the two strains are more than 99\% identical in exon regions. Of 66 genes compared, eight had single nucleotide polymorphisms, and 10 bases out of 33,198 nucleotides (0.03\%) compared were different between two strains in the exon region (data not shown). Next, full-length cDNA sequences were compared with genome sequences comprising each locus and ORF sequences automatically annotated from the genome. The average ORF length translated from cDNA sequences was 559 bases (range, 192-1,506). There were discrepancies between $a b$ initio genome annotation and full-length cDNA in 24 of 83 clones (about 28\%), suggesting incorrect annotation or the possibility of alternative splicing. There were five types of 

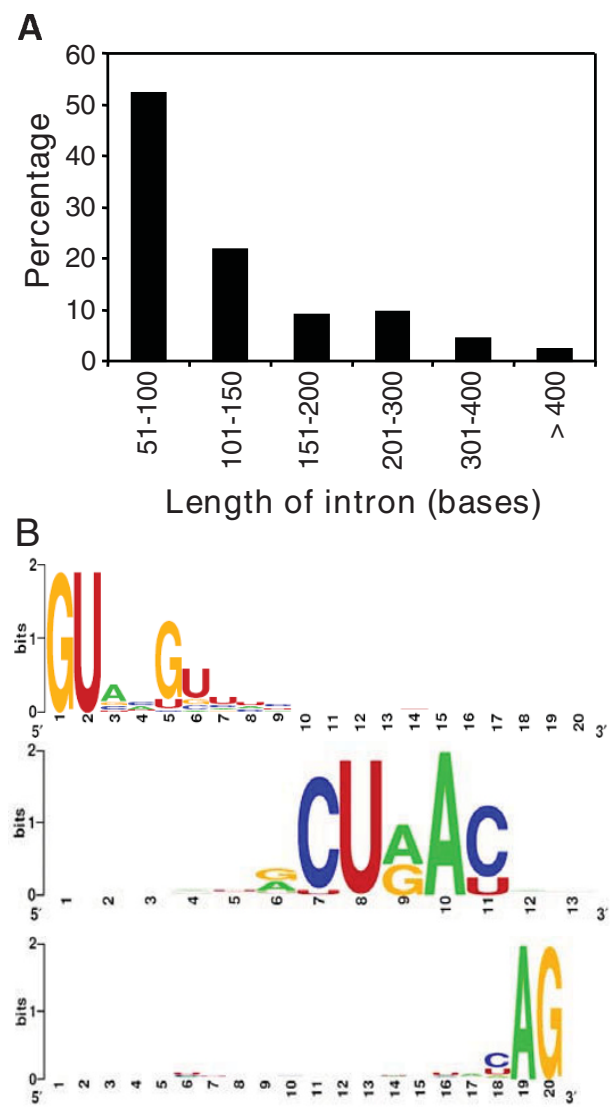

Figure 6 Intron length distribution and splicing site context. (A) Intron length distribution of 68 full-length cDNA sequences. (B) Splicing site consensus sequences for $5^{\prime}$ exon-intron junctions (upper), branch point (middle), and $3^{\prime}$ intron-exon junctions (lower) were calculated using WebLogo server at http://weblogo.berkely. edu. The general consensus sequences of each region were displayed in order of predominance from top to bottom at each position with large letter being higher frequency.

mis-annotation (Fig 5). One group had long N-terminal extensions in the electronic annotation, compared with coding sequences (CDs) inferred from cDNA sequences. In the second group, electronic annotations revealed relatively short CDs in which the start codon resided in an intron or in the middle of coding regions of cDNA sequences. In four cases, the electronically annotated locus had long C-terminal extensions due to erroneous splicing. Another group of loci started at the same site of cDNA, but had another intron in the cDNA, resulting in short ORFs. These sequences did not seem to be the splicing variant between fungal strains, because ESTs from strain 70-15 in which the genome sequence was revealed matched exactly with our cDNA sequences. In these four cases, electronic annotations started and stopped at the exact sites, but depicted other internal exon-intron boundaries. These analyses showed that, in most cases, erroneous splicing was attributed to mis-annotation.
Therefore, the splicing site context of the M.oryzae genome was further analyzed using full-length cDNA sequences.

\section{Splicing site context}

Fungal gene expression, as in higher eukaryotes, accompanies accurate splicing via the coordinated efforts of a spliceosome. Cis elements, consisting of the 5'- and 3'-splice sites, have been found universally to be predominantly GU and AG, respectively, the branch point A and surrounding motif, and polypyrimidine tracts [34]. These fl-cDNA sequences were also used to identify accurate exon-intron boundaries and conserved intron cis elements in M.oryzae. 165 intron sequences from 68 genes were extracted by aligning ESTs to genomic sequences, and these intron sequences with adjacent exon sequences were examined for correct splicing. The mean intron length was 137 nucleotides (range, 53-883, and a dominant peak distribution between 50-100 nucleotides; Fig. 6A). All but one intron examined had the canonical 5'GU...AG3' donor acceptor splicing site pair. The consensus sequences for the region of the 5'-ss, branch site, and 3'-ss are shown as a component of the structure logos in Fig. 6B. The 5'ss consensus sequence was found to be NG|GURMGY, which is more degenerate than NG|GURAGU, a filamentous fungal consensus sequence suggested by Kupfer et al. [34]. The 3'ss consensus sequence was YAG, which is characteristic of metazoans. More than $98 \%$ of the introns appeared to have a potential branch site, a key intron element required for lariat formation during the splicing process, according to icat.pl script. The consensus sequences could be derived to be RCURAY, where the underlined $\mathrm{A}$ is the branch point. This is consistent with the results of Kupfer et al. [34]. The branch point A could be localized to a position between 5 and $160 \mathrm{nt}$ from the 3'-end of the intron, in which most of them (89.7\%) were localized between 11 and 30 nt. Polypyrimidine tracts, another conserved element in mammalian introns, which function as a binding site for spliceosomal protein $\mathrm{U}_{2} \mathrm{AF}^{65}$, were also found in most introns (94\%) screened in this study. About $43 \%$ of them were only located from the 5'ss to the branch point, and $45.4 \%$ were located on both sides of the branch point. These results are also in agreement with Kupfer et al. [34].

\section{Discussion}

Fungal genes expressed during infectious growth within their host and plant genes expressed after attack by their pathogen may have important role(s) in virulence and defense responses. In this study, we identified 2,302 uniESTs expressed during rice- $M$. oryzae interactions using two complementary approaches. The first approach was EST analysis (IL) of blast fungus-infected rice leaves with rapidly expanding typical blast lesions. We harvested leaves with typical lesions and collected 
these daily from 410 days after inoculation. 1,539 uniESTs were identified from the EST analysis. The second strategy was using SSH (SL) to enrich fungal genes and rice genes, whose expression was induced during interactions, resulting in the generation of 963 uniESTs.

\section{Fungal genes expressed during infectious growth were identified}

cDNA libraries were constructed with RNA from rice leaves infected with rice blast fungus. Thus, pathogen genes, as well as host plant genes expressed during their interaction, were contained in these libraries. Assigning the origin of ESTs can be complex. The $\mathrm{G}+\mathrm{C}$ content was successfully used to identify oomycete genes from soybean genes infected with Phytophthora sojae [35]; however, this approach is not applicable for pathosystems involving ascomycete fungi. Sequence similarity to previously identified genes is widely used as a major criterion to distinguish their origin $[7,11,17,19,20]$. However, ESTs that did not show significant sequence similarity to database entries could not be annotated. This ranged from $30 \%$ to more than $40 \%$ of ESTs, depending on the organism. We could not identify the origin of $42.7 \%$ of ESTs in our previous study [7]. Li et al. [19] adapted codon usage pattern as a subsidiary tool to differentiate Sclerotinia sclerotiorum genes from Brassica napus genes. However, rice and $M$. oryzae genes do not show significant differences in their codon usage pattern when we analyzed full-length cDNA sequences (data not shown). In this study, we took advantage of genome sequences and many EST sequences of both organisms to distinguish the origin of the ESTs. With the BLASTX results against NCBI nr, BLASTN data against genomic and EST sequences of both organisms, 99\% of uniESTs could be annotated for their origin (Additional file 1 Table S1). Among these, 712 uniESTs were identified as fungal genes, comprising $31 \%$ of the 2,302 uniESTs. 402 uniESTs (533 ESTs) were from the infected library, comprising $24 \%$ of the uniESTs (27\% of total ESTs), and 310 uniESTs were added from the subtraction library.

A relatively high proportion of ESTs were of fungal origin. This result is consistent with our previous study [7], where about $24.6 \%$ of uniESTs were believed to be fungal genes. Interaction transcriptome [36] approaches have been used for understanding molecular mechanisms of defense and/or pathogenicity in a variety of pathosystems [7,11,17-20,37]. However, only a limited number (or proportion) of fungal genes were identified. This is primarily because previous authors used plant material at early stages of infection, or partially resistant host cultivars. Jantasuriyarat et al. [11] analyzed 68,920 ESTs from eight cDNA libraries including six constructed from rice leaves infected with rice blast fungus, and found only four fungal genes. They collected rice leaves at 6 and $24 \mathrm{~h}$ after blast fungus inoculation, which are the very early stages of infection when fungal conidia have just germinated or just started to penetrate into rice epidermal cells. Talbot et al. [29] estimated fungal biomass to be $10 \%$ at $72 \mathrm{~h}$ after inoculation, at which time lesions start to appear. Recovering a high portion of fungal genes in this study might be attributed to the leaf material from which total RNA was isolated. We collected diseased leaves at late stages of infection daily up to 10 days after inoculation, when the whole leaf blade was covered with expanding lesions and had started to wither. Thus, fungal genes found comprised $27 \%$ of uniESTs in the infection library; this result was not unexpected. These fungal genes will provide a good resource to understand fungal pathogenicity, especially at later stages of infection.

\section{Subtraction efficiency is high}

$43.5 \%$ of fungal genes (310 uniESTs of 712 fungal genes) were obtained from a subtraction library. $50 \%(1,128$ of $2,259)$ of the ESTs from the subtraction library were identified as fungal genes. We used uninfected rice RNA as a driver to enrich fungal genes as well as rice genes that were up-regulated on fungal infection. Gilleroux and Osbourn [20] used two driver cDNA populations (i. e., one derived from mock-inoculated roots and the other from a G. graminis culture grown in complete medium) to subtract cDNAs from G.graminis-infected wheat roots. However, they did not identify any fungal gene using this strategy, possibly because fungal genes comprise a low proportion of infected root tissue and/or the fungal genes expressed during plant infection are also expressed during growth in vitro, to be subtracted out by the fungal driver. Using uninfected plant RNA as a driver turned out to be a useful strategy to identify candidate fungal genes to be functionally characterized. In addition to cataloging fungal genes expressed during infection, we could identify suites of rice genes encoding ABC transporter families specifically obtained from the subtraction library. Forty-two ESTs (18 uniESTs) showed sequence similarity to 11 different ABC transporter families, of which 38 ESTs were from the subtraction library. Seventeen ESTs encoded PDR9, expression of which is induced by environmental stresses, such as heavy metals, hypoxic stress, and redox perturbations [38]. Direct evidence for a role of $\mathrm{ABC}$ transporters in plant defense was obtained from the study of $N p P D R 1$ (formerly named $N p A B C 1$ ), a plasma membrane PDR type ABC transporter of Nicotiana plumbaginifolia, which was induced by the elicitor analog sclareolide, sclareol, a virulent strain of Pseudomonas syringe pv. tabaci and by non-pathogenic pseudomonads, P. fluorescens and P.marginalis pv marginalis [39], 
and is believed to be involved in the secretion of the antimicrobial diterpene, sclareol [40]. Transgenic plants in which NpPDR1 expression was prevented by RNA interference showed reduced resistance to the fungal pathogen Botrytis cinerea [39]. Thus, ABC transporters identified in this study may play a role in rice defense against the invading fungus. These results lead us to suggest that various materials toxic to plant cells might be excreted from invading fungal cells into the plant cytosol, and that plant cells struggle to pump out and/ or detoxify these deleterious materials. This was further supported by the fact that a high proportion $(10.4 \%, 74$ genes) of in planta expressed fungal genes encoded proteins with a signal peptide.

There was little overlap in the fungal genes identified from the two libraries in terms of uniEST. This may be attributed to the low depth of coverage in this study. Another explanation may be drawn if we consider the number of total ESTs, as most overlapped sequences are highly redundant so that 587 ESTs comprised 75 uniESTs.

\section{Novel genes identified}

Although EST collections form the foundation for various genome-scale experiments within as yet unsequenced genomes [33], they provide valuable information for gene discovery, genome annotation, and expression profiles, even in organisms where the genome sequence is available. They have also been successfully applied to identify genes expressed during the interaction of the plant pathogen with its host $[7,35]$. Although 12,465 uniESTs of $M$. oryzae from nine different libraries [12] were already available, we found 100 previously unidentified unigenes through analysis with strict criteria. This exactly identified $14 \%$ of the 712 fungal genes. Sixty seven unigenes were sequenced from the infection library, while 37 genes were added from the subtraction library. The nine libraries that Ebbole et al. [12] used were constructed with RNA covering the fungal life cycle, including conidia, appressoria forming germlings, and mixed culture undergoing mating, which are stress conditions thought to mimic the environment the fungus might encounter in nature, such as rice cell walls, complete medium, minimal medium, nitrogen starvation, and mixed stress conditions, and mycelia from mutants that could not elaborate the infection structure, the appressorium. Nutrient starvation, especially nitrogen-deprived conditions, is regarded as an environmental cue for the fungus to infect the plant $[41,42]$. M. oryzae secrets proteins that cause senescence of rice leaves, reminiscent of the symptoms caused by the fungus itself when it is under nitrogen starvation [42]. NPR1 and NPR2, nitrogen-regulatory genes nonallelic to NUT1, were reported to positively regulate both nitrogen metabolism and pathogenicity [43]. However, many genes were newly identified only from in planta ESTs, indicating that the repertories of genes expressed during infectious growth and under nutrient stress might differ, and that nutrient stress might not fully represent the conditions that the fungus would meet within the plant cell, as suggested in our previous work [7].

\section{Resources for improvement of genome annotation}

ESTs can be used in gene discovery and genome annotation. Of 712 ESTs from this study, 125 (17.5\% of fungal ESTs) did not match any electronically annotated gene. These sequences will help in further correction of genome annotations. Validation of genome annotation could further be confirmed by comparing full-length cDNA sequences with genome sequences. Full-length or near full-length cDNA encompassing the complete ORF can serve as a valuable resource for accurate genome annotation and functional analysis. Large-scale fulllength cDNA sequencing projects with the aim of identifying the complete transcriptome have been conducted and used for improving genome annotation in many model organisms, including Arabidopsis thaliana [44-47] and rice [25], as well as humans [48] and mice $[49,50]$. For example, $32 \%$ of the first version of the Arabidopsis gene model was found to be inaccurately annotated, of 10,507 genes having fl-cDNA sequences, and was improved by incorporation of fl-cDNA sequences [47]. The rice genome community has recognized the value of fl-cDNA sequences and deep EST resources, and acquired more than 28,000 fl-cDNA clones prior to completion of the genome sequence [25]. Few efforts have been made to collect and use full-length cDNA sequences in filamentous fungi, in which targeted gene deletion mutants could readily be obtained by homologous recombination due to the haploid nuclear status. However, full-length cDNA sequences from filamentous fungi still have the same significance for accurate genome annotation, functional analysis, and for proteomics as in other eukaryotes, because fungal genomes are also interspersed with non-coding DNA sequences. In this respect, full-length cDNA clones acquired in this study could be a valuable resource for the community. Of 83 full-length cDNAs, 24 (28.6\%) showed different gene structures from the $a b$ initio annotated gene model. Electronic annotation started at different positions in 11 cases and erroneously stopped in eight cases, resulting in longer or shorter ORFs. In the remaining cases, electronic annotation started and stopped at the exact sites, but depicted erroneous internal exonintron boundaries. In most cases, erroneous splicing was attributed to the wrong gene model. The intron cis elements (i.e., 5'-GU... AG-3' donor-acceptor splicing site pairs, 5' - and 3'-ss 
consensus sequence, internal branch site, and polypyrimidine tracts) were also conserved in the $M$. oryzae genome, and were consistent with results for other filamentous fungi, with more degenerate 5'-splice sites, as suggested by Kupfer et al. [34]. These results can be used to further improve genome annotation.

In summary, genome-wide identification of genes expressed during the interaction between rice and the invading fungal pathogen $M$. oryzae was carried out and resulted in cataloging of 2,302 uniESTs. More than 700 fungal genes were identified, of which 100 genes were newly identified in this study, and more than 80 genes with full-length ORF sequences were used to validate the genome annotation. Further characterization of the genes reported here will help to unravel the mechanisms of pathogenicity, especially at later stages of infection, as well as the defense responses of the host plant.

\section{Conclusions}

Infection of plants by pathogens and the subsequent disease development are accompanied by substantial changes in the physiology of both partners. Analysis of genes expressed during these interactions represents a powerful strategy to obtain insights into the molecular events underlying these changes. Here, we used two complementary strategies, EST and SSH, to catalog genes during a compatible rice and blast fungus interaction at late stages of infection. Fungal genes constituting as many as $30 \%$ of uniESTs, as well as plant defense genes, were identified. We identified 100 previously unreported fungal genes despite the wealth of genome resources on this fungus. Additionally, whole sequences from clones having full-length cDNAs were determined by primer walking, which was used to verify the $a b$ initio annotated gene model of $M$. oryzae genome sequences and splicing context analysis. Taken together, the data obtained in this study will serve as a valuable resource to expand our understanding of genome organization and molecular aspects during rice $M$. oryzae interactions.

\section{Methods}

\section{Construction of cDNA libraries}

Rice plants (Oryza sativa L. cv. Hwacheong) at the $3^{\text {rd }}$ to $4^{\text {th }}$ leaf stages were spray-inoculated with $2 \times 10^{5}$ spores $/ \mathrm{ml}$. After keeping in a dew chamber for $24 \mathrm{~h}$ at $25^{\circ} \mathrm{C}$, the inoculated plants were transferred to the greenhouse to let the lesions develop. Rice leaves on which typical susceptible type lesions spread through the entire leaves were cut and immediately frozen in liquid nitrogen daily up to 10 days after inoculation (in planta sample). Total RNA was isolated from the frozen plant tissues through phenol/chloroform extraction, followed by lithium chloride precipitation [51]. Poly $\mathrm{A}(+)$
RNA was purified using the PolyATrack mRNA isolation system (Promega) and used for construction of two libraries. cDNA was synthesized using the lamda ZAP cDNA synthesis kit and cDNAs larger than $0.5 \mathrm{~kb}$ after gel filtration were used to construct the cDNA library (infection library: IL) using the ZAP Express cDNA Gigapack III Gold Cloning kit (Stratagene, La Jolla, CA, USA). Mass in vivo excision was conducted to rescue phagemid clones. Individual colonies were grown in LB medium amended with $100 \mu \mathrm{g} / \mathrm{ml}$ ampicillin in 96 well plates and used for plasmid preparation. A subtracted cDNA library was also constructed by suppression subtractive hybridization using PCR-select ${ }^{\mathrm{mat}} \mathrm{cDNA}$ subtraction kit (Clontech, Palo Alto, CA, USA) following the manufacturer's instructions. cDNA obtained from $M$. oryzae infected rice leaves was used as a 'tester' and cDNA from uninfected healthy leaves was used as a 'driver'. Subtracted PCR products were cloned into pCR4 blunt-TOPO vector (Invitrogen) and transformed into $E$. coli strain TOP10 (Invitrogen) with blue-white selection. White colonies were grown in LB plates amended with ampicillin and used for plasmid preparation.

\section{EST sequencing and analysis}

Plasmid DNAs were purified from $1 \mathrm{ml}$ each of the overnight grown bacterial cultures and subjected to automated sequencing using ABI PRISM BigDye Terminator on ABI Prism 3700 sequencer (Applied Biosystems, Foster City, CA, USA) or using ET terminator dye (Amersham, Uppsala, Sweden) on the multicapillary sequencer of RISA384 system (Shimadzu, Tokyo, Japan). Sequence analysis was conducted with an automated pipeline embedded in the web-based bioinformatic systems, Comparative Fungal Genomics Platform (http:// cfgp.snu.ac.kr) [52] and Fungal EST Database (FED; http://fedb.snu.ac.kr, Park et al., in preparation). Sequence data in the chromatogram file were processed using Phred [53] to call bases with cutoff value $>20$ and Crossmatch to mask vector sequences. The output sequences were further trimmed using a computer script to get rid of the poly A or poly $\mathrm{T}$ tracks. The sequences longer than $100 \mathrm{bp}$ were collected and clustered using CAP3 [54]. The resulting uniESTs were searched against GenBank nr database using a BLASTX algorithm, and against rice [3,4] and $M$. oryzae [2] genome sequences with the BLASTN algorithm. Functional categorization was conducted with $\mathrm{GO}$ term assignment. Ab initio annotated ORFs from $M$. oryzae genome and the translated rice full-length cDNA sequences, which have corresponding infectionESTs were used to search the InterPro database (version12), and the InterPro terms were mapped to GO terms as described in Ebbole et al. [12]. For full-length cDNA sequences, representing cDNA clones were retrieved and their sequences read 
by primer walking. The intron and exon databases were created by using FELINES [55] with the parameters used by Kupfer et al. [34]. Splicing site consensus sequences for 5' exon-intron junctions, branch point, and 3' intron-exon junctions were calculated using WebLogo server at (http://weblogo.threeplusone.com/) [56]. EST sequences were deposited into dbEST of NCBI under the serial accession number from GT930449 to GT934681, GT968229, and GT968230. The full length cDNA sequences were deposited into NCBI under the serial accession numbers from GU395207 to GU395289 and listed in Table S3.

\section{Real-time RT-PCR}

Real-time RT-PCR was conducted following the protocols described previously [8] with slight modification. Total RNA for cDNA library was used to assess the expression level during infectious growth. Fungal tissue for expression dynamics during development and nutrient starvation was prepared according to the procedures as described previously [8]. Conidia bearing mycelia grown on oatmeal agar for 10 days at $25^{\circ} \mathrm{C}$ were harvested by scraping the surface with razor blade. A total of $2 \times 10^{7}$ conidia were incubated in liquid complete media for 8 hours at $25^{\circ} \mathrm{C}$ by shaking to get germinated conidia. Mycelia for nutrient starvation conditions were prepared as described in Talbot et al. [29]. Samples were frozen in liquid nitrogen immediately after harvest, ground with pre-chilled mortar and pestle, and stored at $-80^{\circ} \mathrm{C}$ before use. Total RNA was isolated from frozen mycelial powder using an Easy-Spin RNA extraction kit (iNtRON Biotechnology, Seoul, Korea). Five micrograms of total RNA was reverse transcribed into first-strand cDNA with oligo (dT) primer using SuperScript ${ }^{\text {th }}$ FirstStrand Synthesis System (Invitrogen, Carlsbad, CA, USA) according to the manufacturer's instruction, and diluted to the concentration of input RNA to be 12.5 $\mathrm{ng} / \mu \mathrm{l}$ with nuclease-free water. Reactions were done in a $10 \mu \mathrm{l}$ volume containing $100 \mathrm{nM}$ of each primer, $2 \mu \mathrm{l}$ cDNA (25 ng of input RNA) and $5 \mu \mathrm{l}$ of $2 \times \mathrm{iQ}^{\mathrm{mm}} \mathrm{SYBR}^{\circ}$ Green Supermix (Bio-rad, Hercules, CA, USA). Realtime PCR was run on an iCycler iQ Real-Time PCR Detection System (Bio-Rad, Hercules, CA, USA). After 3 min denaturation at $95^{\circ} \mathrm{C}$, samples were run for 40 cycles of $15 \mathrm{~s}$ at $95^{\circ} \mathrm{C}, 30 \mathrm{~s}$ at $60^{\circ} \mathrm{C}$, and $30 \mathrm{~s}$ at $72^{\circ} \mathrm{C}$. After each run, amplification specificity was checked with a dissociation curve acquired by heating the samples from 60 to $95^{\circ} \mathrm{C}$. Relative abundance of transcripts in complete media was calculated with $2^{-\Delta \mathrm{Ct}}$, where $\Delta \mathrm{Ct}$ $=\left(C_{t} \text {, gene of interest }-C_{t} \text {, cyclophilin }\right)_{\mathrm{CM}}$. Fold changes during infectious growth and growth under nutrient starvation compared to growing in liquid complete medium were calculated with $2^{-\Delta \Delta \mathrm{Ct}}$, where $\Delta \Delta \mathrm{Ct}=\left(\mathrm{C}_{\mathrm{t}}\right.$, gene of interest ${ }^{-}$ $\mathrm{C}_{\mathrm{t}}$, cyclophilin $)_{\text {test condition }}-\left(\mathrm{C}_{\mathrm{t}}\right.$, gene of interest $-\mathrm{C}_{\mathrm{t}}$, cyclophilin $)$ control [57]. Real-time PCR was conducted with 3 replicates. The primer pairs for transcripts amplification were denoted in Additional file 4 (Table S4).

Additional file 1: List of abundant ESTs sequenced more than $\mathbf{2 0}$
times. List of genes frequently represented in each library.
Click here for file
[http://www.biomedcentral.com/content/supplementary/1471-2164-11-
104-S1.DOC]
Additional file 2: List of genes specific to infection EST. List of novel
genes uniquely or preferentially expressed during rice-M. oryzae
interactions. These were identified by the comparing the publically
available genomic resources as illustrated in Fig. 3.
Click here for file
[http://www.biomedcentral.com/content/supplementary/1471-2164-11-
104-S2.DOC]
Additional file 3: List of genes with full length cDNA. cDNAs with
full-length ORF and their accession number were presented.
Click here for file
[http://www.biomedcentral.com/content/supplementary/1471-2164-11-
104-S3.DOC]
Additional file 4: Primer information for genes used in real-time RT-
PCR. List of primers used in real-time RT-PCR. The expression data was
presented in Fig. 4 .
Click here for file
[http://www.biomedcentral.com/content/supplementary/1471-2164-11-
104-S4.DOC]

Acknowledgements

This work was supported by the National Research Foundation of Korea grants (2009-0063340 and 2009-0080161) and the grants from the Biogreen21 project (20080401-034-044-009-01-00), the TDPAF (309015-04SB020), and the Crop Functional Genomics Center (2009K001198). J. Park is grateful for the graduate fellowship through the Brain Korea 21 Program.

\section{Author details}

'Department of Agricultural Biotechnology, Center for Fungal Pathogenesis, Center for Agricultural Biomaterials and Center for Fungal Genetic Resources, Seoul National University, Seoul 151-921, Korea. ${ }^{2}$ Department of Plant Pathology, The Ohio State University, Columbus, OH 43210, USA.

\section{Authors' contributions}

SK and YH designed the experiments and wrote the manuscript. SK also carried out the experiments and analyzed the data. SP participated in fulllength CDNA sequencing, JP did bioinformatics and developed the database. TKM participated in discussion and manuscript preparation. All authors read and approved the final manuscript.

Received: 4 September 2009

Accepted: 10 February 2010 Published: 10 February 2010

\section{References}

1. Baker B, Zambryski P, Staskawicz B, Dinesh-Kumar SP: Signaling in plantmicrobe interactions. Science 1997, 276:726-733.

2. Dean RA, Talbot NJ, Ebbole DJ, Farman ML, Mitchell TK, Orbach MJ, Thon M, Kulkarni R, Xu JR, Pan H, et al: The genome sequence of the rice blast fungus Magnaporthe grisea. Nature 2005, 434(7036):980-986.

3. Goff SA, Ricke D, Lan TH, Presting G, Wang R, Dunn M, Glazebrook J, Sessions A, Oeller $\mathrm{P}$, Varma $\mathrm{H}$, et al: A draft sequence of the rice genome (Oryza sativa L. ssp. japonica). Science 2002, 296(5565):92-100.

4. Yu J, Hu S, Wang J, Wong GK, Li S, Liu B, Deng Y, Dai L, Zhou Y, Zhang X, et al: A draft sequence of the rice genome (Oryza sativa L. ssp. indica). Science 2002, 296(5565):79-92.

5. Jeon J, Park SY, Chi MH, Choi J, Park J, Rho HS, Kim S, Goh J, Yoo S, Park JY, et al: Genome-wide functional analysis of pathogenicity genes in the rice blast fungus. Nat Genet 2007, 39(4):561-565. 
6. Ahn IP, Kim S, Kang S, Suh SC, Lee YH: Rice Defense Mechanisms Against Cochliobolus miyabeanus and Magnaporthe grisea Are Distinct. Phytopathology 2005, 95(11):1248-1255.

7. Kim S, Ahn IP, Lee YH: Analysis of genes expressed during riceMagnaporthe grisea interactions. Mol Plant-Microbe Interact 2001 14(11):1340-1346.

8. Kim S, Ahn IP, Rho HS, Lee YH: MHP1, a Magnaporthe grisea hydrophobin gene, is required for fungal development and plant colonization. Mol Microbiol 2005, 57(5):1224-1237.

9. Kim S, Ahn IP, Park CH, Park SG, Park SY, Jwa NS, Lee YH: Molecular characterization of the CDNA encoding an acidic isoform of PR-1 protein in rice. Mol Cells 2001, 11(1):115-121.

10. Park CH, Kim S, Park JY, Ahn IP, Jwa NS, Im KH, Lee YH: Molecular characterization of a pathogenesis-related protein 8 gene encoding a class III chitinase in rice. Mol Cells 2004, 17(1):144-150.

11. Jantasuriyarat C, Gowda M, Haller K, Hatfield J, Lu G, Stahlberg E, Zhou B, $\mathrm{Li} \mathrm{H}, \mathrm{Kim} \mathrm{H}, \mathrm{Yu}$ Y, et al: Large-scale identification of expressed sequence tags involved in rice and rice blast fungus interaction. Plant Physiol 2005, 138(1):105-115.

12. Ebbole DJ, Jin $Y$, Thon M, Pan H, Bhattarai E, Thomas T, Dean R: Gene discovery and gene expression in the rice blast fungus, Magnaporthe grisea: analysis of expressed sequence tags. Mol Plant-Microbe Interact 2004, 17(12):1337-1347.

13. Gowda M, Jantasuriyarat C, Dean RA, Wang GL: Robust-LongSAGE (RLSAGE): a substantially improved LongSAGE method for gene discovery and transcriptome analysis. Plant Physiol 2004, 134(3):890-897.

14. Irie T, Matsumura H, Terauchi R, Saitoh H: Serial Analysis of Gene Expression (SAGE) of Magnaporthe grisea: genes involved in appressorium formation. Mol Genet Genomics 2003, 270(2):181-9.

15. Lu JP, Liu TB, Lin FC: Identification of mature appressorium-enriched transcripts in Magnaporthe grisea, the rice blast fungus, using suppression subtractive hybridization. FEMS Microbiol Lett 2005, 245(1):131-137.

16. Rauyaree P, Choi WB, Fang EG, Blackmon B, Dean RA: Genes expressed during early stages of rice infection with the rice blast fungus Magnaporthe grisea. Mol Plant Pathol 2001, 2(6):347-354.

17. Keon J, Antoniw J, Rudd J, Skinner W, Hargreaves J, Hammond-Kosack K Analysis of expressed sequence tags from the wheat leaf blotch pathogen Mycosphaerella graminicola (anamorph Septoria tritici). Fungal Genet Biol 2005, 42(5):376-389.

18. Kruger WM, Pritsch C, Chao S, Muehlbauer GJ: Functional and comparative bioinformatic analysis of expressed genes from wheat spikes infected with Fusarium graminearum. Mol Plant-Microbe Interact 2002, 15(5):445-455.

19. Li R, Rimmer R, Buchwaldt L, Sharpe AG, Seguin-Swartz G, Coutu C, Hegedus DD: Interaction of Sclerotinia sclerotiorum with a resistant Brassica napus cultivar: expressed sequence tag analysis identifies genes associated with fungal pathogenesis. Fungal Genet Biol 2004, 41(8):735-753.

20. Guilleroux M, Osbourn A: Gene expression during infection of wheat roots by the 'take-all' fungus Gaeumannomyces graminis. Mol Plant Pathol 2004, 5(3):203-216.

21. Broeker K, Bernard F, Moerschbacher BM: An EST library from Puccinia graminis $\mathrm{f}$. $\mathrm{sp}$. tritici reveals genes potentially involved in fungal differentiation. FEMS Microbiol Lett 2006, 256(2):273-281.

22. Diatchenko L, Lau YF, Campbell AP, Chenchik A, Moqadam F, Huang B, Lukyanov S, Lukyanov K, Gurskaya N, Sverdlov ED, et al: Suppression subtractive hybridization: a method for generating differentially regulated or tissue-specific CDNA probes and libraries. Proc Natl Acad Sci USA 1996, 93(12):6025-6030.

23. Shearer $\mathrm{G} \mathrm{Jr}$ : Cloning and analysis of CDNA encoding an elongation factor 1 alpha from the dimorphic fungus Histoplasma capsulatum. Gene 1995, 161(1):119-123.

24. Pariasca JAT, Sunaga A, Miyazaki T, Hisaka H, Sonoda M, Nakagawa $H$, Sato T: Cloning of CDNAs encoding senescence-associated genes, ACC synthase and ACC oxidase from stored snow pea pods (Pisum sativum L. var saccharatum) and their expression during pod storage. Postharvest Biol and Technol 2001, 22:239-247.

25. Kikuchi S, Satoh K, Nagata T, Kawagashira N, Doi K, Kishimoto N, Yazaki J, Ishikawa M, Yamada H, Ooka H, et al: Collection, mapping, and annotation of over 28,000 cDNA clones from japonica rice. Science 2003, 301(5631):376-379.

26. Hugot K, Riviere MP, Moreilhon C, Dayem MA, Cozzitorto J, Arbiol G, Barbry P, Weiss C, Galiana E: Coordinated regulation of genes for secretion in tobacco at late developmental stages: association with resistance against oomycetes. Plant Physiol 2004, 134(2):858-870.

27. Oh IS, Park AR, Bae MS, Kwon SJ, Kim YS, Lee JE, Kang NY, Lee S, Cheong H, Park OK: Secretome analysis reveals an Arabidopsis lipase involved in defense against Alternaria brassicicola. Plant Cell 2005, 17(10):2832-2847.

28. Ahn N, Kim S, Choi W, Im KH, Lee YH: Extracellular matrix protein gene, $E M P 1$, is required for appressorium formation and pathogenicity of the rice blast fungus, Magnaporthe grisea. Mol Cells 2004, 17(1):166-173.

29. Talbot NJ, Ebbole DJ, Hamer JE: Identification and characterization of $M P G 1$, a gene involved in pathogenicity from the rice blast fungus Magnaporthe grisea. Plant Cell 1993, 5(11):1575-1590.

30. Bendtsen JD, Nielsen $\mathrm{H}$, von Heijne G, Brunak S: Improved prediction of signal peptides: SignalP 3.0. J Mol Biol 2004, 340(4):783-795.

31. Katagiri T, Shinozaki K: Disruption of a gene encoding phosphatidic acid phosphatase causes abnormal phenotypes in cell growth and abnormal cytokinesis in Saccharomyces cerevisiae. Biochem Biophys Res Commun 1998, 248(1):87-92

32. van Schooten B, Testerink C, Munnik T: Signalling diacylglycerol pyrophosphate, a new phosphatidic acid metabolite. Biochim Biophys Acta 2006, 1761(2):151-159.

33. Rudd S: Expressed sequence tags: alternative or complement to whole genome sequences?. Trends Plant Sci 2003, 8(7):321-329.

34. Kupfer DM, Drabenstot SD, Buchanan KL, Lai H, Zhu H, Dyer DW, Roe BA, Murphy JW: Introns and splicing elements of five diverse fungi. Eukaryot Cell 2004, 3(5):1088-1100.

35. Qutob D, Hraber PT, Sobral BWS, Gijzen M: Comparative analysis of expressed sequences in Phytophthora sojae. Plant Physiol 2000, 123:243-253

36. Birch PR, Kamoun S: Studying interaction transcriptomes: coordinated analyses of gene expression during plant-microorganism interactions. New Technologies for Life Sciences: A Trends Guide New York: Elsevier ScienceWood R 2000, 77-82.

37. Randall TA, Dwyer RA, Huitema E, Beyer K, Cvitanich C, Kelkar H, Fong AM, Gates K, Roberts S, Yatzkan E, et al: Large-scale gene discovery in the oomycete Phytophthora infestans reveals likely components of phytopathogenicity shared with true fungi. Mol Plant-Microbe Interact 2005, 18(3):229-243.

38. Moons A: Ospdr9, which encodes a PDR-type ABC transporter, is induced by heavy metals, hypoxic stress and redox perturbations in rice roots. FEBS Lett 2003, 553(3):370-376.

39. Stukkens Y, Bultreys A, Grec S, Trombik T, Vanham D, Boutry M: NpPDR1, a pleiotropic drug resistance-type ATP-binding cassette transporter from Nicotiana plumbaginifolia, plays a major role in plant pathogen defense. Plant Physiol 2005, 139(1):341-352.

40. Jasinski M, Stukkens Y, Degand H, Purnelle B, Marchand-Brynaert J, Boutry M: A plant plasma membrane ATP binding cassette-type transporter is involved in antifungal terpenoid secretion. Plant Cell 2001, 13(5):1095-1107.

41. Snoeijers SS, P'erez-Garc'ýa A, Joosten MHAJ, Wit PJGMD: The effect of nitrogen on disease development and gene expression in bacterial and fungal plant pathogens. Eur J Plant Pathol 2000, 106:493-506.

42. Talbot NJ, McCafferty HRK, Ma M, Moore K, Hamer JE: Nitrogen starvation of the rice blast fungus Magnaporthe grisea may act as an environmental cue for disease symptom expression. Physiol Mol Plant Pathol 1997, 50(3):179-195.

43. Lau G, Hamer JE: Regulatory genes controlling MPG1 expression and pathogenicity in the rice blast fungus Magnaporthe grisea. Plant Cell 1996, 8(5):771-781.

44. Castelli V, Aury JM, Jaillon O, Wincker P, Clepet C, Menard M, Cruaud C, Quetier F, Scarpelli C, Schachter V, et al: Whole genome sequence comparisons and "full-length" cDNA sequences: a combined approach to evaluate and improve Arabidopsis genome annotation. Genome Res 2004, 14(3):406-413.

45. Haas BJ, Volforsky N, Town CD, Troukhan M, Alexandrov N, Feldmann KA, Flavell RB, White O, Salzberg SL: Full-length messenger RNA sequences greatly improve genome annotation. Genome Biol 2002, 3(6): RESEARCH0029. 
46. Seki M, Narusaka M, Kamiya A, Ishida J, Satou M, Sakurai T, Nakajima M, Enju A, Akiyama K, Oono Y, et al: Functional annotation of a full-length Arabidopsis cDNA collection. Science 2002, 296(5565):141-145.

47. Yamada K, Lim J, Dale JM, Chen H, Shinn P, Palm CJ, Southwick AM, Wu HC, Kim C, Nguyen M, et al: Empirical analysis of transcriptional activity in the Arabidopsis genome. Science 2003, 302(5646):842-846.

48. Imanishi T, Itoh T, Suzuki Y, O'Donovan C, Fukuchi S, Koyanagi KO, Barrero RA, Tamura T, Yamaguchi-Kabata Y, Tanino M, et al: Integrative annotation of 21,037 human genes validated by full-length CDNA clones. PLOS Biol 2004, 2(6):e162.

49. Hayashizaki Y: RIKEN mouse genome encyclopedia. Mech Ageing Dev 2003, 124(1):93-102.

50. Okazaki Y, Furuno M, Kasukawa T, Adachi J, Bono H, Kondo S, Nikaido I, Osato N, Saito R, Suzuki H, et al: Analysis of the mouse transcriptome based on functional annotation of 60,770 full-length CDNAs. Nature 2002, 420(6915):563-573

51. Davis KR, Ausubel FM: Characterization of elicitor-induced defense responses in suspension-cultured cells of Arabidopsis. Mol Plant-Microbe Interact 1989, 2:363-368.

52. Park J, Park B, Jung K, Jang S, Yu K, Choi J, Kong S, Kim S, Kim H, Kim JF, et al: CFGP: a web-based, comparative fungal genomics platform. Nucleic Acids Res 2008, 36(36 Database):562-571.

53. Ewing B, Green P: Base-calling of automated sequencer traces using Phred. II. Error probabilities. Genome Res 1998, 8(3):186-194.

54. Huang X, Madan A: CAP3: A DNA sequence assembly program. Genome Res 1999, 9(9):868-877.

55. Drabenstot SD, Kupfer DM, White JD, Dyer DW, Roe BA, Buchanan KL, Murphy JW: FELINES: a utility for extracting and examining EST-defined introns and exons. Nucleic Acids Res 2003, 31(22):e141.

56. Crooks GE, Hon G, Chandonia JM, Brenner SE: WebLogo: a sequence logo generator. Genome Res 2004, 14(6):1188-1190.

57. Livak KJ, Schmittgen TD: Analysis of relative gene expression data using

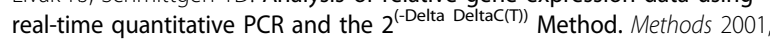
25(4):402-408.

doi:10.1186/1471-2164-11-104

Cite this article as: Kim et al:: Identification and analysis of in planta expressed genes of Magnaporthe oryzae. BMC Genomics 2010 11:104.

\section{Submit your next manuscript to BioMed Central and take full advantage of:}

- Convenient online submission

- Thorough peer review

- No space constraints or color figure charges

- Immediate publication on acceptance

- Inclusion in PubMed, CAS, Scopus and Google Scholar

- Research which is freely available for redistribution 